\title{
PROPAGACIÓN DE PULSOS ÓPTICOS A TRAVÉS DE AMPLIFICADORES DE FIBRA DOPADA CON ERBIO (EDFA)
}

\author{
Fideromo Saavedra G. ${ }^{1} \quad$ Dante Leonelli Z. $^{2} \quad$ Álvaro Lamas N. ${ }^{2}$ \\ Recibido el 10 de Marzo de 2005, aceptado el 26 de octubre de 2005
}

\begin{abstract}
RESUMEN
En el presente artículo se exponen los aspectos más generales de la caracterización del amplificador de fibra dopada con Erbio (EDFA), con el fin de proporcionar una base teórica para la comprensión de los distintos fenómenos que se producen en él, al ser utilizado como medio físico para la amplificación y propagación de pulsos ópticos y así determinar cómo se ven afectados dichos pulsos, debido a la aparición de distintos fenómenos no lineales.

Palabras clave: EDFA, propagación, amplificación, pulsos ópticos.
\end{abstract}

\begin{abstract}
In the present paper the most general aspects of the characterization of the Erbium Doped Fiber Amplifier (EDFA) are exposed; with the purpose of providing a theoretical base for the understanding of the different phenomena that take place inside of the amplifier when it's used like physical medium for the amplification and propagation of optical pulses and thus to determine how these pulses are affected, due to the appearance of different dispersive and nonlinear phenomena.
\end{abstract}

Keywords: EDFA, propagation, amplification, optical pulses.

\section{INTRODUCCIÓN}

En los últimos 20 años la fibra óptica se ha posicionado como el medio de transmisión más importante para las comunicaciones de alta velocidad, tanto digitales como analógicas. Sus características insuperables en comparación con otros medios de transmisión, como son su gran ancho de banda $(\sim 23 \mathrm{THz})$ y su baja atenuación ( $\sim 0.23 \mathrm{~dB} / \mathrm{km}$ en $1550 \mathrm{~nm})$, ha hecho de las fibras ópticas un medio ideal para transmisiones de larga distancia. En la década de los 90 , paralelamente a la creciente necesidad de aumentar el ancho de banda disponible aparecieron en escena los amplificadores ópticos, los cuales dieron paso a las comunicaciones como se conocen hoy en día. En el transcurso de los primeros años de esa década se popularizó la utilización de los amplificadores de fibra dopada con Erbio (EDFA) [3], [7], los cuales poseen la característica principal de entregar máxima ganancia en la región de $1.55 \mu \mathrm{m}$, lo que dio paso a la utilización de la infraestructura ya instalada alrededor del mundo, pero esta vez operando en la $3^{\text {a }}$ ventana y haciendo uso de fibra que había sido diseñada para tener dispersión mínima en $1.31 \mu \mathrm{m}$ [10]. Lo anterior dio paso a nuevos inconvenientes como son el manejo de la dispersión y la no linealidad del medio dieléctrico producto del aumento de la potencia. Producto de esto, es necesario realizar un tratamiento generalizado del problema de la propagación de pulsos a través de los EDFAs, mediante el uso de aproximaciones para las ecuaciones de tasa, en el cual el amplificador es modelado como un sistema atómico de dos niveles. En éste, la respuesta dinámica de dicho sistema atómico es gobernada por el tiempo de relajación de la población $\mathrm{T}_{1}$ y por el tiempo de relajación del dipolo $\mathrm{T}_{2}$. Dicho modelo tiene validez para pulsos ópticos cuyo ancho $\mathrm{T}_{0}$ cumple con la relación $\mathrm{T}_{1}>\mathrm{T}_{0}>\mathrm{T}_{2}$. A partir de lo anterior, y considerando que se trabaja con múltiples longitudes de onda, es posible obtener ecuaciones que rigen la propagación de pulsos a través del amplificador EDFA, tomando en cuenta la contribución que realizan los dopantes y como afecta esta última a la aparición de los fenómenos de dispersión y no linealidad. Además, se considera el efecto no sintonizante que ocurre cuando la

1 Académico Departamento de Ingeniería Eléctrica, Universidad de Santiago de Chile, Casilla 10233, C. Central, Santiago-Chile, fsaavedr@lauca.usach.cl.

2 Alumnos Magíster de Ingeniería Eléctrica, Universidad de Santiago de Chile, Santiago-Chile. 
frecuencia de la portadora de los pulsos de entrada no coincide con la frecuencia donde ocurre el peak de ganancia [6].

\section{CONCEPTOS BÁSICOS}

En los sistemas de comunicación de larga distancia, que hacen uso de la fibra óptica como medio físico para la propagación de las señales ópticas, se tiene que las señales experimentan una atenuación considerable al propagarse por la fibra. Lo anterior obliga a que los sistemas de transmisión utilicen algún mecanismo de amplificación para que las señales mantengan un nivel de potencia detectable por el receptor, para lo cual en la actualidad se hace uso de amplificadores ópticos, como es el caso del amplificador de fibra dopada con Erbio, EDFA.

La idea básica que está detrás de la amplificación en los amplificadores ópticos es la emisión estimulada, que es el mismo principio con que operan los láseres, con la diferencia que en el caso de los amplificadores ópticos no se trabaja con retroalimentación. El principio de operación que presentan los amplificadores ópticos es el siguiente: la fibra dopada con erbio, al ser estimulada mediante una señal de bombeo, causa que los átomos absorban fotones, quedando éstos en estado excitado durante un intervalo que está definido por el tiempo de vida de los átomos de Erbio. Al volver a su estado original, liberan la energía almacenada, fenómeno que es conocido como emisión. La emisión puede presentarse en dos formas distintas: la primera de ellas es la emisión espontánea, donde la principal característica que presenta es que no existe una relación de fase entre los fotones emitidos y los fotones incidentes. La emisión estimulada, en cambio, se inicia producto de la incidencia de un fotón, con lo cual los fotones emitidos coinciden con los fotones incidentes, tanto en fase como dirección, lo que produce una interferencia constructiva, que finalmente produce la amplificación de las señales de luz incidente.

Para que llegue a producirse el fenómeno de emisión dentro del amplificador óptico, debe producirse una inversión de población a nivel de estados de energía, que se traduce en que la cantidad de iones en estado excitado sea mayor que la cantidad de iones en el estado básico de energía. Dicha inversión de población se logra inyectando potencia a la fibra dopada y puede ser realizada mediante un bombeo externo.

En la actualidad el amplificador de fibra dopada con Erbio es el más utilizado entre los amplificadores de fibra dopada, dada las características intrínsecas que posee el Erbio, como es la de operar en la zona cercana a 1.55 $\mu \mathrm{m}$, región en la cual la fibra monomodo utilizada en la actualidad posee mínimas pérdidas. Otras características que presenta el EDFA son su elevada ganancia y bajo ruido, además de poseer un amplio ancho de banda, transparencia a las longitudes de onda e independencia a la polarización, lo que en consecuencia lo hace tremendamente atractivo para su utilización en los sistemas de comunicaciones ópticas.

\section{Modelamiento Físico del EDFA}

El principio de la amplificación, como se mencionó anteriormente, se basa principalmente en las transiciones de energía que sufren los electrones, producto de la absorción o emisión de energía por parte del átomo al cual pertenecen. Una forma de visualizar el principio de operación de un EDFA, puede ser a través de un esquema de bombeo de tres niveles, el cual se muestra en la Fig. 1 [1].

Cuando se inyecta una señal de bombeo a la fibra dopada, los fotones incidentes son absorbidos por los iones de Erbio, provocando de esta forma una transición de electrones desde el nivel básico (nivel $\mathrm{E}_{3}$ ), al nivel de energía superior (nivel $\mathrm{E}_{1}$ ). Tomando en cuenta que la vida media en el nivel $\mathrm{E}_{3}$ no posee una extensa duración $(\approx 1 \mu \mathrm{s})$, en comparación con la vida media del nivel metaestable (nivel $\left.\mathrm{E}_{2}\right)(\sim 10 \mathrm{~ms})$, se tiene que los electrones decaerán al nivel de energía metaestable, a través de una transición no radiativa. En cambio, dada la extensa duración del tiempo de vida media que posee el nivel metaestable, si la señal de bombeo se mantiene en el tiempo, se llegará a producir una inversión de la población entre el nivel de energía metaestable $\mathrm{E}_{2} \mathrm{y}$ el nivel de energía básico $\mathrm{E}_{3}$.

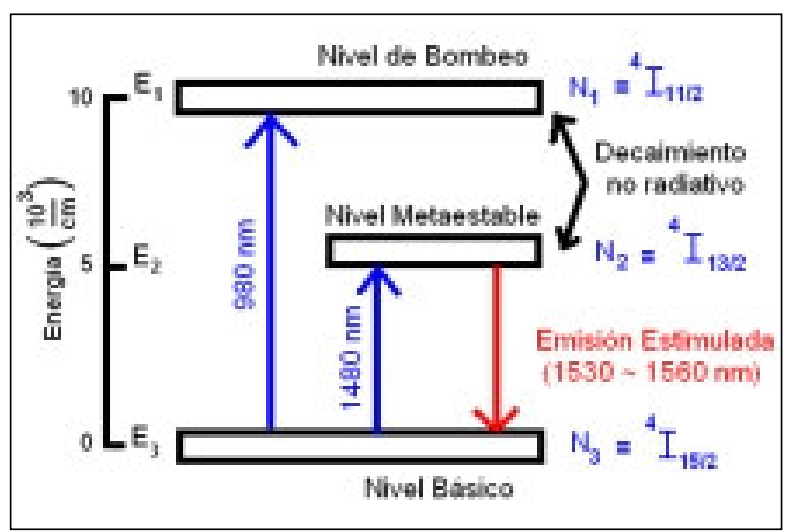

Fig. 1 Representación esquemática del modelo de un EDFA.

Es a partir de esa energía almacenada que se produce la amplificación mediante la emisión estimulada. Sin embargo, además de producirse la emisión estimulada, también se produce el fenómeno de emisión espontánea, producto de que algunos fotones decaen en forma 
espontánea desde el nivel metaestable al nivel básico; por lo cual dicha emisión espontánea es también amplificada, lo que origina el ruido de emisión espontánea o ASE (Amplified Spontaneous Emision). Este efecto, además de introducir ruido a la señal que se propaga, dado que disminuye la población en el nivel metaestable, también hace disminuir la ganancia que proporciona el EDFA. La expresión general de la ganancia está dada por $\mathrm{g}=\sigma\left(\mathrm{N}_{1}-\mathrm{N}_{2}\right)$, donde $\sigma$ corresponde a la sección de cruce de transición y $\mathrm{N}_{1}$ y $\mathrm{N}_{2}$ corresponden a las densidades atómicas de los niveles $E_{1} y E_{2}$, respectivamente.

Como se ve en la Fig. 1, la transición de importancia, que es donde se produce la emisión estimulada, se establece entre los niveles básicos y metaestable. Para el caso particular de utilizar un láser de bombeo en 980 $\mathrm{nm}$, los fotones de bombeo incidentes, correspondientes al bombeo cuya energía corresponde a la diferencia entre los niveles ${ }^{4} I_{11 / 2}$ y ${ }^{4} I_{15 / 2}$, son absorbidos por las transiciones electrónicas, con lo que se excitan los iones de Erbio ubicándolos en el nivel ${ }^{4} I_{11 / 2}$. Por lo tanto, a partir de ese nivel, los electrones decaen espontáneamente, dada la corta duración del tiempo de vida $\tau_{32}$ (del orden de los microsegundos, en comparación con el tiempo de vida $\tau_{21} \sim 10 \mathrm{~ms}$ ), a través de transiciones no radiativas, hasta el nivel metaestable ${ }^{4} I_{13 / 2}$. Si el bombeo continúa, la población $\mathrm{N}_{2}$ correspondiente al nivel metaestable ${ }^{4} I_{13 / 2}$, llegará a ser mayor que la población $\mathrm{N}_{1}$ correspondiente al nivel básico ${ }^{4} I_{15 / 2}$, produciéndose la inversión de la población necesaria para producir la amplificación de los fotones correspondientes a la señal ubicada en la región de fluorescencia alrededor de $1530 \mathrm{~nm}$. La corta duración del tiempo de vida $\tau_{32}$ de los fotones en el nivel de bombeo, permite simplificar el modelo del EDFA a un modelo de sólo dos niveles, correspondientes al nivel básico y al nivel metaestable, sin pérdida de exactitud.

\section{PROPAGACIÓN DE PULSOS ÓPTICOS A TRAVÉS DEL EDFA}

La propagación de pulsos ópticos a través de la fibra óptica monomodo bajo condiciones de dispersión y no linealidad ha sido estudiada ampliamente [4]. Por lo que para poder llevar a cabo el estudio de la propagación de pulsos ópticos a través de los EDFAs es necesario realizar algunas modificaciones con el fin de incluir el efecto que producen los dopantes, a través de la modificación de la polarización total inducida $P(\vec{r}, t)$, la que esta dada por la expresión [1] - [3]:

$$
\vec{P}(\vec{E})=\vec{P}^{L}(\vec{E})+\vec{P}^{N L}(\vec{E})
$$

la cual debe incluir un tercer término $P_{d}(\vec{r}, t)$ el que representa la contribución de los dopantes, que a su vez modifica la constante dieléctrica, debido a la contribución que introduce la susceptibilidad del sistema atómico de dos niveles que modela el comportamiento del EDFA.

Tomando como punto de partida las ecuaciones de Maxwell, es posible obtener la siguiente ecuación [5], [6], [7] y [9]:

$$
\nabla \times \nabla \times \vec{E}=-\mu_{0} \varepsilon_{0} \frac{\partial^{2} \vec{E}}{\partial t^{2}}-\mu_{0} \frac{\partial^{2} \vec{P}}{\partial t^{2}}-\mu_{0} \sigma \frac{\partial \vec{E}}{\partial t}
$$

Para el caso particular de dos campos ópticos propagándose en diferentes longitudes de onda, cuyas frecuencias centrales están dadas por $\omega_{1}$ y $\omega_{2}$, se tiene que el campo eléctrico $E(\vec{r}, t)$ puede ser escrito como:

$$
\begin{aligned}
E(\vec{r}, t)=\frac{\vec{e}_{x}}{2}[ & E_{1} e^{j\left(\omega_{1} t-\beta_{1} z\right)}+E_{1}^{*} e^{j\left(\omega_{1} t-\beta_{1} z\right)} \\
& \left.+E_{2} e^{j\left(\omega_{2} t-\beta_{2} z\right)}+E_{2}^{*} e^{j\left(\omega_{2} t-\beta_{2} z\right)}\right]
\end{aligned}
$$

$\vec{e}_{x}$ es la dirección de polarización, que en este caso se asume invariante y a su vez perpendicular a $\vec{e}_{z}, \beta_{1}$ y $\beta_{2}$ son las constantes de propagación a las frecuencias $\omega_{1} \mathrm{y}$ $\omega_{2}$, respectivamente.

Para tomar en cuenta la contribución que realizan los dopantes, es necesario modificar la ecuación (1), con lo cual la nueva expresión para la polarización inducida estará dada por:

$$
\vec{P}(\vec{E})=\vec{P}^{L}(\vec{E})+\vec{P}^{N L}(\vec{E})+\vec{P}_{d}(\vec{E})
$$

Tomando en cuenta que la polarización corresponde a la contribución lineal, o sea, en este caso un medio isotrópico y considerando el caso para dos campos eléctricos, se tiene que la expresión que define a la polarización lineal esta dada por:

$$
P_{M}^{L}(\vec{r}, t)=\varepsilon_{0} \int_{-\infty}^{\infty} \aleph^{L}(\tau)\left(E_{M 1}(\vec{r}, t-\tau)+E_{M 2}(\vec{r}, t-\tau)\right) d \tau
$$

De igual manera, para el caso de dos campos eléctricos propagándose, la expresión para la polarización no lineal, puede ser escrita como:

$$
\begin{aligned}
P^{N L}=\varepsilon_{0} X^{N L}[ & E_{1}(\vec{r}, t) \cdot e^{j\left(\omega_{1} t-\beta_{1} z\right)}+E_{1}^{*}(\vec{r}, t) \cdot e^{j\left(\omega_{1} t-\beta_{1} z\right)} \\
& \left.+E_{2}(\vec{r}, t) \cdot e^{j\left(\omega_{2} t-\beta_{2} z\right)}+E_{2}^{*}(\vec{r}, t) \cdot e^{j\left(\omega_{2} t-\beta_{2} z\right)}\right]^{3}
\end{aligned}
$$


Por lo tanto, desarrollando la ecuación anterior, es posible obtener dos expresiones que gobiernan las contribuciones de la polarización no lineal para las frecuencias $\omega_{1}$ y $\omega_{2}$, respectivamente [8]:

$$
\begin{aligned}
P_{1}^{N L}(\vec{r}, t) & =\vec{e}_{x}\left|P_{1}^{N L}\right| \cos \left(\omega_{1} t-\beta_{1} z\right) \\
& =\frac{\vec{e}_{x}}{2}\left[P_{1}^{N L} e^{j\left(\omega_{1} t-\beta_{1} z\right)}+P_{1}^{* N L} e^{-j\left(\omega_{1} t-\beta_{1} z\right)}\right] \\
& =\varepsilon_{0} X^{N L} \frac{3}{4}\left[\left|E_{1}\right|^{2}+2\left|E_{2}\right|^{2}\right] E_{1} \\
P_{2}^{N L}(\vec{r}, t) & =\vec{e}_{x}\left|P_{2}^{N L}\right| \cos \left(\omega_{2} t-\beta_{2} z\right) \\
& =\frac{\vec{e}_{x}}{2}\left[P_{2}^{N L} e^{j\left(\omega_{2} t-\beta_{2} z\right)}+P_{2}^{* N L} e^{-j\left(\omega_{2} t-\beta_{2} z\right)}\right] \\
& =\varepsilon_{0} X^{N L} \frac{3}{4}\left[\left|E_{2}\right|^{2}+2\left|E_{1}\right|^{2}\right] E_{2}
\end{aligned}
$$

Para el caso de la polarización producto de la contribución de los dopantes, se tiene que su expresión para el caso de dos campos eléctricos, estará dada por [1], [6]:

$$
P_{d}(\vec{r}, t)=\varepsilon_{0} \int_{-\infty}^{\infty} \chi_{d}(\tau)\left(E_{M 1}(\vec{r}, t-\tau)+E_{M 2}(\vec{r}, t-\tau)\right) d \tau
$$

donde la susceptibilidad $\chi_{d}(\omega)$ para un sistema atómico de dos niveles, se define en frecuencia como [6]:

$$
\chi_{d}(\omega)=\frac{g_{p} c}{\omega} \frac{\left(\omega-\omega_{a}\right) T_{2}-j}{1+\left(\omega-\omega_{a}\right)^{2} T_{2}^{2}}
$$

donde $\omega$ es la frecuencia óptica de la señal propagante, $\omega_{\mathrm{a}}$ es la frecuencia de resonancia atómica y $\mathrm{g}_{\mathrm{p}}$ se define como el peak de ganancia $g_{p}=\sigma\left(N_{1}-N_{2}\right)$. Aquí $\sigma$ corresponde a la sección de cruce de transición, y $N_{l} \mathrm{y}$ $\mathrm{N}_{2}$ corresponden a las densidades atómicas.

Mediante un trabajo algebraico y después de la aplicación de la transformada de Fourier es posible obtener la siguiente ecuación:

$$
\begin{aligned}
\operatorname{RotRot}( & \left.E_{M 1}\left(\vec{r}, \omega-\omega_{01}\right) e^{-j \beta_{01} z}\right)^{j \beta_{01} z}= \\
& \left(\frac{\omega}{c}\right)^{2} E_{M 1}\left(\vec{r}, \omega-\omega_{01}\right)-j \xi \omega E_{M 1}\left(\vec{r}, \omega-\omega_{01}\right) \\
+ & \left(\frac{\omega}{c}\right)^{2} X^{L}(\omega) E_{M 1}\left(\vec{r}, \omega-\omega_{01}\right)-X_{d}(\omega) E_{M 1}\left(\vec{r}, \omega-\omega_{01}\right) \\
+ & \frac{3}{4}\left(\frac{\omega}{c}\right)^{2} X^{N L}\left(\left|E_{M 1}(\vec{r}, t)\right|^{2}+2\left|E_{M 2}(\vec{r}, t)\right|^{2}\right) E_{M 1}\left(\vec{r}, \omega-\omega_{01}\right)
\end{aligned}
$$

A partir de la ecuación anterior y de manera análoga a lo realizado en el segundo párrafo, es posible definir una constante dieléctrica que en este caso considera el efecto de la susceptibilidad $X_{d}$ Esta expresión está dada por [6]:

$$
\begin{aligned}
\varepsilon(\omega)=1+X^{L} & (\omega)+X_{d}(\omega) \\
& +\frac{3}{4} X^{N L}(\omega)\left[\left|E_{1}\right|^{2}+2\left|E_{2}\right|^{2}\right]
\end{aligned}
$$

Mediante esta nueva consideración, la ecuación (10) asume la forma:

$$
\begin{aligned}
\operatorname{RotRot} & \left(E_{M 1}\left(\vec{r}, \omega-\omega_{01}\right) e^{-j \beta_{01} z}\right) e^{j \beta_{01} z}= \\
& =\left(\frac{\omega}{c}\right)^{2}[\varepsilon(\omega)-j \xi \omega] E_{M 1}\left(\vec{r}, \omega-\omega_{01}\right)
\end{aligned}
$$

Si se aplica un cambio de variable de la forma $E_{M}(\vec{r}, \Delta \omega)=F(x, y) \tilde{A}(z, \Delta \omega)$, es posible obtener dos ecuaciones donde la primera de ellas describe la distribución transversal del campo, la cual, a su vez, permite determinar la constante de propagación. En cambio, la segunda ecuación representa la evolución de la envolvente de la onda a través de su propagación en el eje $z$, donde dichas ecuaciones están dadas por:

$$
\begin{aligned}
& \left(\frac{\partial^{2}}{\partial x^{2}}+\frac{\partial^{2}}{\partial y^{2}}\right) F_{1}(x, y)+\left[\left(\frac{\omega}{c}\right)^{2} \varepsilon(\omega)-\beta_{1}^{2}(\omega)\right] F_{1}(x, y) \\
& \left(\left(\frac{\partial^{2}}{\partial z^{2}}-j 2 \beta_{01} \frac{\partial}{\partial z}\right)+\left[\beta_{1}^{2}(\omega)-\beta_{01}^{2}-j \omega \xi\right]\right) A_{1}(z, \Delta \omega)
\end{aligned}
$$

Debido a la complejidad introducida por la componente no lineal de la constante dieléctrica (ecuación 11), es necesario aplicar la teoría de perturbación de primer orden [2], [3] y [5]. Así es posible obtener expresiones tanto para la distribución modal $F_{1}(\mathrm{x}, \mathrm{y})$, donde dicha constante corresponde a la distribución modal del modo fundamental propagado; como así también para la constante de propagación. Primeramente se obtiene la distribución modal $F_{1}(\mathrm{x}, \mathrm{y})$ y la constante de propagación $\beta_{1}(\omega)$, a partir de considerar solamente la componente lineal de la constante dieléctrica, es decir:

$$
\varepsilon(\omega)=1+X^{L}(\omega)+X_{d}(\omega)
$$

En esta ecuación, $\varepsilon(\omega)$ incluye el efecto de los dopantes a través de $X_{d}(\omega)$. Luego se obtiene la constante de propagación total a partir de considerar la componente no lineal de la constante dieléctrica como una pequeña perturbación de la solución lineal. No se afecta la 
distribución modal $F_{1}(\mathrm{x}, \mathrm{y})$, pero si al valor propio $\beta_{1}(\omega)$. De lo anterior y mediante la expansión en serie de Taylor, tanto de la constante dieléctrica como también de la susceptibilidad atómica $X_{\mathrm{d}}$ y utilizando la transformada inversa de Fourier, es posible obtener:

$$
\begin{gathered}
\frac{\partial a_{1}(z, t)}{\partial z}+\beta_{11}^{e f f} \frac{\partial a_{1}(z, t)}{\partial t}-j \frac{\beta_{12}^{e f f}}{2} \frac{\partial^{2} a_{1}(z, t)}{\partial t^{2}}+\frac{\alpha}{2} a_{1}(z, t) \\
-\left(\frac{g_{p} c}{2 n_{01}\left(\omega_{01}\right)} \frac{\delta-1}{1+\delta}\right) a_{1}(z, t)+j \gamma_{1}\left[p_{01}\left|a_{1}(z, t)\right|^{2}\right. \\
\left.+2 p_{02}\left|a_{2}(z, t)\right|^{2}\right] a_{1}(z, t)+a_{s p}=0
\end{gathered}
$$

con

$$
\begin{aligned}
& \beta_{11}^{e f f}=\beta_{11}+\frac{g_{p}}{2 n_{01}} \frac{1+2 j \delta-\delta^{2}}{\left(1+\delta^{2}\right)^{2}} T_{2} \\
& \beta_{12}^{e f f}=\frac{\beta_{12}}{2}+\frac{g_{p}}{2 n_{01}} \frac{\delta\left(\delta^{2}-3\right)+j\left(1+3 \delta^{2}\right)}{\left(1+\delta^{2}\right)^{3}} T_{2}^{2} \\
& \gamma_{1}=\frac{n_{2}\left(\omega_{01}\right) \omega_{01}}{A_{e f f} c} ; n_{2}\left(\omega_{01}\right)=\frac{3 X^{N L}}{8 n_{L}\left(\omega_{01}\right)}, \\
& A_{e f f}=\frac{\left(\int_{-\infty}^{\infty} \int_{-\infty}^{\infty}\left|F_{1}(x, y)\right|^{2} d x d y\right)^{2}}{\int_{-\infty}^{\infty} \int_{-\infty}^{\infty}\left|F_{1}(x, y)\right|^{4} d x d y} \text { y } a_{1}=\frac{\xi \omega_{01}}{\beta_{01}} .
\end{aligned}
$$

El término $P 01$ corresponde a la potencia máxima, la cual se utiliza con el fin de determinar la potencia instantánea distribuida en la sección transversal para una fibra a una longitud $\mathrm{z}$ cualquiera, mediante la aplicación de la siguiente ecuación:

$$
\begin{aligned}
p(z, t) & =\int_{-\infty}^{\infty} \int_{-\infty}^{\infty}\left|E_{M}(\vec{r}, t)\right|^{2} d x d y \\
& =|A(z, t)|^{2} \int_{-\infty}^{\infty} \int_{-\infty}^{\infty}|F(x, y)|^{2} d x d y=p_{0} p_{n}(z, t)
\end{aligned}
$$

donde $p_{n}(z, t)$ se define como la potencia instantánea normalizada a su valor máximo $P_{0}$ en una posición $z$, con lo que se normaliza la amplitud de pulso $a(z, t)$ tal que $p_{n}(z, t)=|a(z, t)|^{2}$. De esta forma se llega a la siguiente expresión, para la obtención de la ecuación (16) [7]:

$$
A(z, t)=\frac{\sqrt{P_{0} a(z, t)}}{\sqrt{\left(\int_{-\infty}^{\infty} \int_{-\infty}^{\infty}|F(x, y)|^{2} d x d y\right)}}
$$

El termino $a_{\mathrm{sp}}$ de la ecuación (16) corresponde a la contribución debido al ruido ASE.

De manera análoga a lo obtenido para la señal óptica propagándose a la frecuencia $\omega_{1}$, es decir, la ecuación (16), es posible extrapolar una expresión para la señal óptica propagándose a la frecuencia $\omega_{2} \mathrm{u}$ otra frecuencias, mediante el uso de la siguiente expresión:

$$
\begin{aligned}
& \frac{\partial a_{i}(z, t)}{\partial z}+\beta_{i 1}^{e f f} \frac{\partial a_{i}(z, t)}{\partial t}-j \frac{\beta_{i 2}^{e f f}}{2} \frac{\partial^{2} a_{i}(z, t)}{\partial t^{2}} \\
& +j \gamma_{i}\left[p_{0 i}\left|a_{i}(z, t)\right|^{2}+2 p_{0 k} \sum_{k \neq i}^{M}\left|a_{2}(z, t)\right|^{2}\right] a_{i}(z, t) \\
& -\left(\frac{g_{p} c}{2 n_{0 i}\left(\omega_{0 i}\right)} \frac{\delta-1}{1+\delta}\right) a_{i}(z, t)+\frac{\alpha}{2} a_{i}(z, t)+a_{s p i}=0
\end{aligned}
$$

Donde los parámetros se definen de igual forma que para la ecuación (16).

\section{Observaciones}

Como puede verse en la ecuación (20), aparecen dos términos que son incógnitas relativas a la operación intrínseca del EDFA, como es la ganancia $g_{p}$ y el término $a_{\mathrm{sp}}$ asociado al ruido ASE, los cuales es necesario calcularlos de alguna forma para poder incluirlos en el modelo obtenido. Se puede utilizar el modelamiento clásico del EDFA [3], mediante el cual es posible obtener el siguiente sistema de ecuaciones [10]:

$$
\begin{gathered}
\frac{d P_{p}^{ \pm}(z)}{d z}=\mp P_{p}^{ \pm}(z)\left(\alpha_{13} \frac{\bar{n}_{1}}{\bar{n}_{E r}}\right) \mp \ell_{p} P_{p}^{ \pm}(z) \\
\frac{d P_{s}\left(z, v_{i}\right)}{d z}=P_{s}\left(z, v_{i}\right)\left(g_{21, i} \frac{\bar{n}_{2}}{\bar{n}_{E r}}-\alpha_{12, i} \frac{\bar{n}_{1}}{\bar{n}_{E r}}\right)-\ell_{i} P_{s}\left(z, v_{i}\right)
\end{gathered}
$$

donde $i=1,2, \ldots \ldots, \mathrm{N}$. 


$$
\begin{aligned}
\frac{d P_{A S E}{ }^{ \pm}\left(z, v_{j}\right)}{d z} & = \pm P_{A S E}{ }^{ \pm}\left(z, v_{j}\right)\left(g_{21, j} \frac{\bar{n}_{2}}{\bar{n}_{E r}}-\alpha_{12, j} \frac{\bar{n}_{1}}{\bar{n}_{E r}}\right) \\
+ & 2 h v_{j} \Delta v_{j} g_{21, j} \frac{\bar{n}_{2}}{\bar{n}_{E r}}+\ell_{j} P_{A S E}^{ \pm}\left(z, v_{j}\right)
\end{aligned}
$$

donde $j=1,2, \ldots .$, M.

con $\mathrm{I}_{\mathrm{i}, \mathrm{j}}$ pérdidas de la fibra para las señales y ASE, $\mathrm{I}_{\mathrm{p}}$ pérdidas de la fibra para el bombeo, $\alpha_{12} \mathrm{y} \mathrm{g}_{21}$ coeficientes de absorción y ganancia de las señales y ASE y $\alpha_{13}$ coeficiente de absorción del bombeo. $\mathrm{N}$ corresponde a la cantidad de canales WDM que ingresan al sistema y $\mathrm{M}$ al número de ranuras de frecuencia en que es dividido el espectro óptico. Asimismo, los signos ( \pm ) corresponden al sentido de propagación de las señales ópticas. Además el sistema presentado anteriormente posee las siguientes condiciones de borde:

$$
\begin{gathered}
P_{p}^{+}(0)=P_{p}^{-}(L)=P_{p 0} \\
P_{s}\left(0, v_{i}\right)=P_{s 0}\left(v_{i}\right) \\
P_{A S E}^{+}\left(0, v_{j}\right)=P_{A S E}^{-}\left(L, v_{j}\right)=0
\end{gathered}
$$

\section{CONCLUSIONES}

En el presente trabajo se han expuesto los aspectos más importantes que se utilizan al momento de caracterizar el problema de la propagación de pulsos a través de amplificadores de fibra dopada con Erbio. Mediante el uso de aproximaciones para las ecuaciones de tasa, el amplificador es modelado como un sistema atómico de dos niveles, donde la respuesta dinámica de dicho sistema atómico es gobernada por el tiempo de relajación de población $\left(T_{1}\right)$ y por el tiempo de relajación del dipolo $\left(T_{2}\right)$. A partir de lo anterior, considerando que se trabaja con múltiples longitudes de onda, se desarrollaron las ecuaciones que rigen la propagación de pulsos a través del amplificador EDFA. Se tomó en cuenta la contribución que realizan los dopantes y cómo afecta dicha contribución a la aparición de los fenómenos de dispersión y no linealidad, como son la automodulación de fase (SPM) y modulación de fase cruzada (XPM). Además del efecto desintonizante que ocurre, cuando la frecuencia de la portadora de los pulsos de entrada no coincide con la frecuencia donde ocurre el peak de ganancia.

Los sistemas de ecuaciones aquí expuestos permiten la realización de un programa computacional que permitirá la simulación de la propagación de ondas ópticas a lo largo de un amplificador EDFA, bajo diferentes regímenes de trabajo.

\section{AGRADECIMIENTOS}

Este trabajo fue realizado en el marco del proyecto FONDEF DOOI1026, "Redes Ópticas para Internet del futuro".

\section{REFERENCIAS}

[1] G.P. Agrawal. "Aplication of Nonlinear Fiber Optics". Elsevier Sience. Primera edición. California, Estados Unidos. 2001.

[2] G.P. Agrawal. "Nonlinear Fiber Optics". Elsevier Science. Tercera edición. California, Estados Unidos. 2001.

[3] R. Giles y E. Desurvire, "Modeling erbium-doped fiber amplifiers". J. Lightwave Technol. Vol. 9, pp. 271-283. February 1991.

[4] F. Saavedra. "Sistemas de transmisión por fibra óptica”. Universidad de Santiago de Chile. 1996.

[5] S. Reichel, W. Lenz y R. Zengerle. "Simulation of phase modulation in EDFA's using an extended model". IEEE Photon. Technol. Lett. Vol. 10, pp. 1724-1726. December 1998.

[6] G.P. Agrawal. "Optical pulse propagation in doped fiber amplifiers". Physical Review A. Vol. 44, 1, pp. 781-789. December 1991.

[7] S. Reichel, R. Zengerle. "Effects of Nonlinear Dispersion in EDFA's on Optical Comunication System”. J. Lightwave Technol. Vol. 17, pp. 11521157. July 1999.

[8] B. Jiménez. "Fenómeno de mezcla de cuatro ondas en sistemas WDM". Tesis para optar al grado de Master. Departamento Ingeniería Eléctrica. Universidad de Santiago de Chile. Santiago, Chile. 2001.

[9] L.O. Jaraquemada. "Dispersión y automodulación de fase en fibra óptica monomodo en régimen no lineal". Tesis para optar al grado de Magíster. Departamento Ingenería Eléctrica. Universidad de Santiago de Chile. Santiago, Chile. 2000.

[10] R. Olivares. "Estudio de transientes de potência e de técnicas de controle em redes WDM baseadas em cascatas de amplificadores a fibra dopada com 
Rev. Fac. Ing. - Univ. Tarapacá, vol. 13 No 3, 2005

Érbio (EDFAs)". Tesis para optar al grado de doctor.

Departamento de Engenharia Eléctrica. Pontificia

Universidade Católica do Rio de Janeiro. 2000. 